\title{
Survivin, a key component of the Wnt/ $\beta$-catenin signaling pathway, contributes to traumatic brain injury-induced adult neurogenesis in the mouse dentate gyrus
}

\author{
LIN ZHANG $^{1,2^{*}}$, RONG YAN ${ }^{1,2 *}$, QI ZHANG ${ }^{1}$, HAINING WANG ${ }^{1}$, XIAOKUI KANG ${ }^{1}$, JIA LI $^{1}$, \\ SHUYUAN YANG ${ }^{1}$, JIANNING ZHANG ${ }^{1}$, ZHENLIN LIU $^{2}$ and XINYU YANG ${ }^{1}$ \\ ${ }^{1}$ Department of Neurosurgery, Tianjin Medical University General Hospital, Tianjin Neurological Institute, \\ Tianjin Key Laboratory of Injuries, Variations and Regeneration of Nervous System, Tianjin 300052; \\ ${ }^{2}$ Department of Neurosurgery, Tianjin 5th Central Hospital, Tianjin 300450, P.R. China
}

Received April 6, 2013; Accepted July 8, 2013

DOI: $10.3892 / \mathrm{ijmm} .2013 .1456$

\begin{abstract}
The enhancement of endogenous neurogenesis has been suggested in the treatment of traumatic brain injury (TBI); however, the factors that trigger the process of adult neurogenesis following TBI remain elusive. In the adult mammalian central nervous system, there are 2 neurogenic regions: the subgranular zone (SGZ) of the hippocampus and the subventricular zone (SVZ) of the lateral ventricles, both of which maintain relatively quiescent states in a stable microenvironment. However, once stimulated by intrinsic and extrinsic events, relevant signals are activated in these 2 regions. In this study, in order to explore the mechanisms behind endogenous neurogenesis following TBI, we investigated potential factors regulating this process. We observed that the expression of survivin, an anti-apoptotic protein, increased in a time-dependent manner in the hippocampus in a mouse model of TBI. In addition, the number of survivin (+) cells, as well as that of BrdU (+) cells increased in the SGZ of the dentate gyrus (DG) in the hippocampus following TBI, as shown by immunofluorescence double staining; the co-localization of survivin and BrdU was shown in the merged images. The expression of survivin was also significantly increased in the doublecortin (DCX) $(+)$ immature neurons in the DG of the hippocampus soon after the induction of TBI. Taken together, these data confirm the connection between the expression of survivin and adult neurogenesis following TBI;
\end{abstract}

Correspondence to: Dr Xinyu Yang, Department of Neurosurgery, Tianjin Medical University General Hospital, 154 Anshan Road, Heping District, Tianjin 300052, P.R. China

E-mail: yangxinyu@tijmu.edu.cn

Dr Zhenlin Liu, Department of Neurosurgery, Tianjin 5th Central Hospital, 41 Zhejiang Road, Tanggu District, Tianjin 300450, P.R. China E-mail: wjzhenlin817@sina.com

\section{${ }^{*}$ Contributed equally}

Key words: survivin, dentate gyrus, adult neurogenesis, traumatic brain injury our data also suggest the therapeutic potential of upregulating survivin expression as a novel strategy for the effective treatment of TBI.

\section{Introduction}

Traumatic brain injury (TBI) is a major cause of mortality and morbidity globally $(1,2)$, and continues to be an important public health issue worldwide $(3,4)$. Appropriate treatment is vital for patient survival and the restoration of nervous function following TBI. The concept of enhancing endogenous neurogenesis and replacing lost neurons following TBI is now becoming a reality (4). It is widely accepted that new neurons are generated in the adult mammalian brain throughout life. In the adult central nervous system (CNS), active neurogenesis occurs in 2 discrete 'neurogenic' regions: the subgranular zone (SGZ) of the dentate gyrus (DG) in the hippocampus and the subventricular zone (SVZ) of the lateral ventricles in the forebrain $(5,6)$. Neurogenesis in these regions maintains relatively quiescent states in a stable microenvironment. However, studies have indicated that neurogenesis can be induced by TBI (7-9) and increased levels of cell proliferation have been observed in the DG and SVZ soon after TBI $(7,10,11)$. Traumatic insult to the brain results in neural tissue injury through cell-death processes, including apoptosis or programmed cell death. Therefore, the processes of promoting the proliferation of neural stem cells and the differentiation of newly generated neurons may play a role in the enhancement of endogenous neurogenesis to replace injured neural cells following TBI.

The Wnt/ $\beta$-catenin signaling pathway is important for neurogenesis in the developing nervous system $(12,13)$, as well as in adults, which consists of an active process encompassing proliferation, migration, differentiation and synaptogenesis (14). Thus, increased $\beta$-catenin signaling following TBI (15) may contribute to repair deterioration and may promote the recovery of neural function. In this study, we wished to explore the mechanisms behind adult neurogenesis induced by Wnt/ $\beta$-catenin signaling following TBI. We aimed to determine whether survivin, the downstream target gene of the Wnt/ $\beta$-catenin signaling pathway $(16,17)$, 
plays a role in the enhancement of neurogenesis following TBI.

Survivin, a member of the inhibitor of apoptosis (IAP) gene family (18), is expressed in the G2/M phase of the cell cycle in a cycle-regulated manner. Survivin is also a regulator of mitosis, and is localized to mitotic spindle microtubules (19) in a specific and saturable reaction that is regulated by microtubule dynamics (20), to promote mitosis and cell proliferation. Evidence indicates that disrupting the survivin-microtubule interaction results in the loss of the anti-apoptotic function of survivin (19). Survivin plays a role in maintaining cell viability at mitosis, potentially also controlling apoptosis, thus regulating cell division. Unlike other IAP proteins, survivin plays a dual role as an apoptotic inhibitor and as a chromosomal passenger protein (CPP) (21). In addition, survivin binds to and inhibits the activity of caspase-3, caspase-7 and caspase-9 (22-25), which are involved in cell death during mitosis. Most importantly, survivin is crucial for normal embryonic neurogenesis $(26,27)$. It is noteworthy that survivin is ubiquitously expressed during embryonic development, and diffusely expressed in the developing nervous system (28), but only minimally expressed in adult tissues; in contrast, it is widely expressed in human malignant tumors. Furthermore, studies have indicated that survivin also plays a role in certain physiological processes, as well as in pathological conditions, such as TBI (29). The expression of survivin may attenuate DNA cleavage, in a cell-specific manner, following TBI (30). These findings prompted us to investigate the correlation between the expression of survivin and adult neurogenesis following TBI. In this study, we investigated the expression of survivin at certain time points following TBI and detected variations in the expression of survivin in several types of existing nerve cell in the DG of the hippocampus using a mouse model of TBI induced by lateral fluid percussion.

\section{Materials and methods}

A total of 146 adult male C57BL/6 mice (10 weeks of age; 25-30 g) obtained from the Academy of Military Medical Sciences, China, were housed in cages under a 12-h light-dark cycle with a regular food and water supply. All experimental procedures were approved by the China Small Animal Protection Association (CSAPA). For the study, the mice were randomly divided into a TBI group and a sham-operated group.

Lateral fluid percussion model of TBI. Each mouse was anesthetized by an intraperitoneal injection of $10 \%$ chloral hydrate (4 $\mu \mathrm{l} / \mathrm{g})$, placed in a stereotactic apparatus and the dorsal scalp was exposed through a midline cut under sterile conditions. A $3 \mathrm{~mm}$ craniotomy was made on the right parietal bone, between the lambda and bregma suture and $1 \mathrm{~mm}$ lateral to the sagittal suture. A Luer lock fitting was cemented to the skull as previously described (31). The TBI group was subjected to lateral fluid percussion injury of $202 \pm 2 \mathrm{kPa}$ using a pre-calibrated fluid percussion injury device. The sham-operated group was subjected to identical surgery without lateral fluid percussion. Following injury, the Luer lock fitting was removed, the wound was sutured and the mice were returned to their cages.

Quantitative polymerase chain reaction. Animals with TBI were sacrificed at designated times (12 h, 1, 2, 5, 7 and 14 days post
TBI) (each time point, $n=6$ ). The sham-operated animals were sacrificed on day 3 post surgery $(n=6)$. Hippocampal tissues from the ipsilateral hemispheres were rapidly excised and 'snap-frozen' with liquid nitrogen. The primers used for PCR were as follows: survivin forward, 5'-TACCGCATCGCCA CCTTC-3' and reverse, 5'-CCAAATCAGGCTCGTTCTCG-3'; and GAPDH forward, 5'-AGGTCGGTGTGAACGGA TTTG-3' and reverse, 5'-TGTAGACCATGTAGTTGA GGTCA-3'. Total RNA was isolated from the samples using TRIzol reagent (Cat\# GMRS-001, GenePharma, Shanghai, China). cDNA synthesis was performed using $1 \mathrm{mg}$ of total RNA with the mRNA Selective PCR Kit (AMV) version 1.1 (Takara Biotechnology, Dalian, China) according to the manufacturer's instructions. Quantitative PCR was performed using the SYBR ${ }^{\circledR}$ Premix Ex Taq ${ }^{\mathrm{TM}}$ (TaKaRa) in combination with $0.5 \mu \mathrm{l}$ primers in the MX3000P ${ }^{\mathrm{TM}}$ Real-Time PCR Instrument (Stratagene, La Jolla, CA, USA). All PCR reactions were performed using standard PCR conditions: stage $1: 95^{\circ} \mathrm{C}$ for $3 \mathrm{~min}(1 \mathrm{cycle})$; stage $2: 95^{\circ} \mathrm{C}$ for $12 \mathrm{sec}$, followed by $62^{\circ} \mathrm{C}$ for $40 \mathrm{sec}$; stage 3 : from $62-95^{\circ} \mathrm{C}$, followed by $0.2^{\circ} \mathrm{C}$ for $2 \mathrm{sec}$ (1 cycle). The controls were consistently found to be negative for survivin. PCR products were resolved on a $1.5 \%$ agarose gel in Tris-acetate buffer and visualized by ethidium bromide staining under UV illumination.

Western blot analysis. To further detect the protein expression of survivin in the hippocampus, the animals in the 2 groups were sacrificed at $12 \mathrm{~h}, 1,2,5,7$ and 14 days following TBI or sham operation (each time point, $n=6$ ) and ipsilateral hippocampal tissues were immediately 'snap-frozen' in liquid nitrogen. Each hippocampus was homogenized in ice-cold homogenizing buffer with a sonicator. Homogenized tissues were centrifuged at $12,000 \mathrm{rpm}$ for $10 \mathrm{~min}$ at $4^{\circ} \mathrm{C}$. Following electrophoretic separation on a $10 \%$ SDS-PAGE gel, the resolved proteins were electrophoretically transferred onto a PVDF membrane (Millipore, Billerica, MA, USA). The membranes were incubated in blocking buffer for $2 \mathrm{~h}$ at room temperature and probed with an anti-survivin antibody (1:1,000 dilution; Abcam, Cambridge, MA, USA) and an antibody against $\beta$-actin $(1: 1,000$ dilution; Cell Signaling Technology, Inc., Danvers, MA, USA) overnight at $4^{\circ} \mathrm{C}$. The goat anti-rabbit secondary antibody conjugated to horseradish peroxidase (HRP) were then applied for $2 \mathrm{~h}$ at room temperature. Bound antibodies were detected by an enhanced chemiluminescence assay (SuperSignal ${ }^{\mathrm{TM}}$ West Pico Chemiluminescent Substrates, Pierce Biotechnology, Inc., Rockford, IL, USA).

5'-Bromo-2'-deoxyuridine (BrdU) administration. The animals received intraperitoneal injections of BrdU (Sigma-Aldrich, St. Louis, MO, USA; $100 \mathrm{mg} / \mathrm{kg}$; dissolved in saline to a final concentration of $20 \mathrm{mg} / \mathrm{ml}$ ). Injections were administered twice daily on days 1 and 2, followed by a single injection on day 3 following brain injury. The animals were sacrificed $2 \mathrm{~h}$ after the final BrdU injection. The BrdU-labeled newborn neurons were counted within 3 days after administration (from day 1 to 3 post TBI) .

Tissue preparation and immunofluorescence staining. For histological evaluation, the animals were perfused with cold phosphate-buffered saline (PBS) followed by $4 \%$ parafor- 


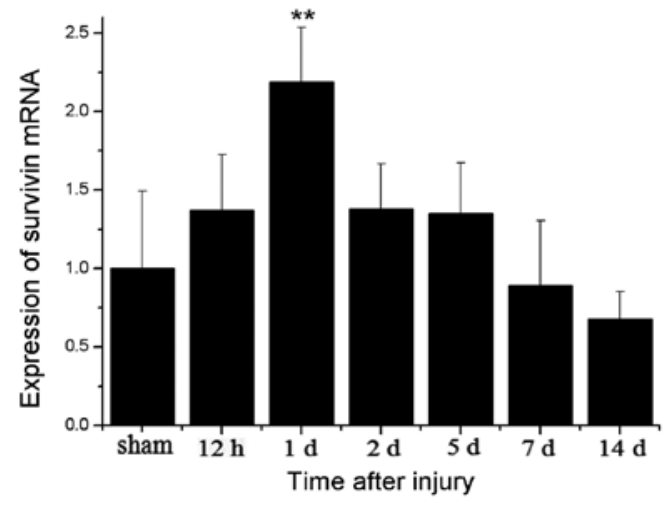

Figure 1. Increased mRNA expression of survivin induced by traumatic brain injury (TBI). Ipsilateral hippocampal tissues were removed and total RNA was isolated at $12 \mathrm{~h}, 1,2,5,7$ and 14 days after TBI. Data are presented as relative levels of survivin in the hippocampus to the sham-operated group; each TBI time point and sham operation represents data as the means \pm SEM values $(\mathrm{n}=6){ }^{* *} \mathrm{p}<0.01$ versus sham-operated group.

maldehyde solution under deep anesthesia. The brains were removed, kept in $4 \%$ paraformaldehyde for $24 \mathrm{~h}$ after fixation and immersed in $30 \%$ sucrose for $24-48 \mathrm{~h}$ at $4^{\circ} \mathrm{C}$. The frozen brains were cut coronally in $35-\mu$ m-thick slices on a cryostat throughout the rostrocaudal extent of the hippocampus and the slices were collected in 6-well plates filled with PBS. In order to assess the number of positive cells, 1-in-5 series of sections from each animal were processed for immunostaining as previously described by Wojtowicz and Kee (32). Briefly, to eliminate non-specific background, the sections were incubated in PBS blocking solution containing $0.3 \%$ Triton X-100, 2\% goat serum in $0.1 \mathrm{M}$ PBS for $60 \mathrm{~min}$ at $37^{\circ} \mathrm{C}$ prior to staining. The sections were then incubated with BrdU/doublecortin (DCX)/glial fibrillary protein (GFAP) $/ \mathrm{NeuN}$ and survivin primary antibody diluted in blocking solution at $4^{\circ} \mathrm{C}$ overnight on a shaker. The primary antibodies used in this study were rat anti-BrdU (1:50 dilutions, Santa Cruz Biotechnology, Inc., Santa Cruz, CA, USA), mouse anti-DCX (1:100 dilution; Santa Cruz Biotechnology), mouse anti-GFAP (1:200 dilution; Millipore), mouse anti-NeuN (1:100 dilution; Millipore) and rabbit anti-survivin (1:400 dilution; Cell Signaling Technology). Finally, the sections were incubated with fluorochrome-conjugated secondary antibody in the dark, diluted in $0.1 \mathrm{M}$ PBS (at $\mathrm{pH} 7.4$ ) with $0.3 \%$ Triton X solution for $1 \mathrm{~h}$ at $37^{\circ} \mathrm{C}$. The fluorescent secondary antibodies were Alexa Fluor 555 goat anti-rabbit IgG (1:1000 dilutions, Cell Signaling Technology), Alexa Fluor 488 goat anti-mouse IgG (1:1000 dilutions, Cell Signaling Technology) and Alexa Fluor 488 goat anti-rat IgG (1:1,000 dilutions, Cell Signaling Technology). The slices for the negative control of survivin staining were subjected to identical staining without primary antibody. Fluorescence images were acquired using an inverted Olympus fluorescence microscope and an image capture system.

Quantification for immunofluorescence results. All analyses were accomplished with stereological counting methods. A systematic random sampling of 1-in-5 series of coronal sections was prepared from each animal and processed for immunofluorescence staining. The hippocampus (bregma -1.3 to $-3.1 \mathrm{~mm}$ ) was selected to prepare the sections. The
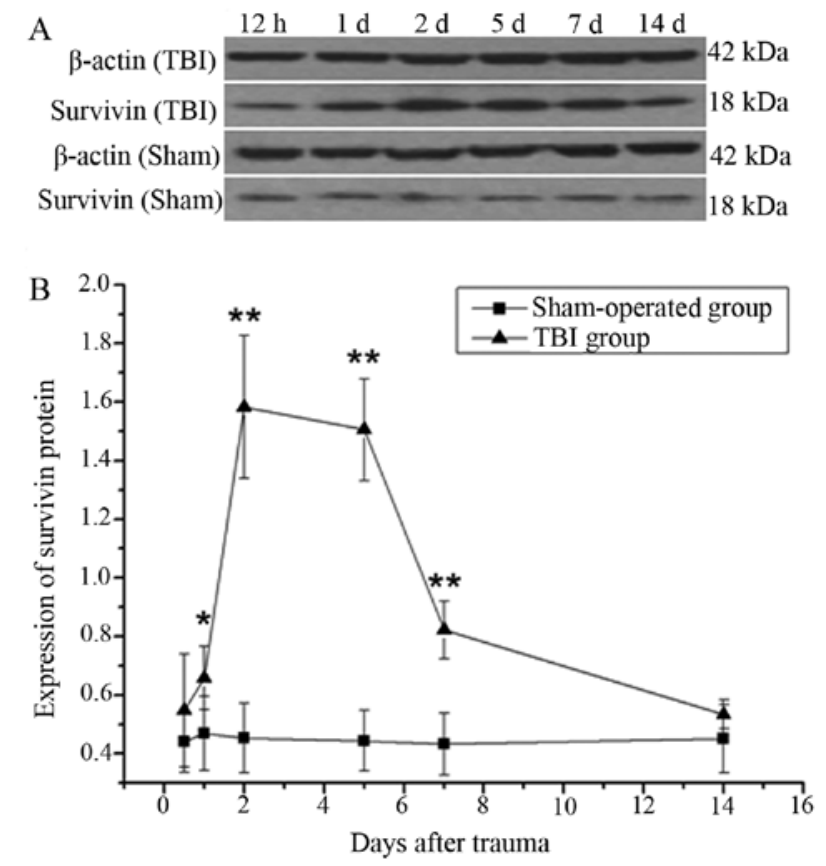

Figure 2. Increased protein expression of survivin in ipsilateral hippocampus after traumatic brain injury (TBI). As shown by western blot analysis, survivin expression increased in a time-dependent manner. (A) Densitometric analysis of survivin bands in ipsilateral hippocampus after TBI shows significant increases in a time-dependent manner. (B) Expression of survivin in ipsilateral hippocampus of TBI group and sham-operated group at $12 \mathrm{~h}, 1$, $2,5,7$ and 14 days post model set up. The measurement of $\beta$-actin was used as an internal control. The expression of survivin was quantified by a semiquantitative densitometry. Data are represented as the means \pm SEM values $(n=6)$. ${ }^{*} \mathrm{p}<0.05$ versus sham-operated group; ${ }^{* *} \mathrm{p}<0.01$ versus sham-operated group.

entire SGZ of the DG was assessed and each survivin-labeled cell was examined to assess the co-labeling of survivin with cell-type-specific markers. Every positive cell in the SGZ of the DG was counted using a $x 40$ objective lens. The percentage of double-labeled cells was calculated as the number of cells that were stained with both survivin and a given cell-type-specific marker against the total number of survivin-positive cells. Four animals in each group with 10 sections per brain were examined. The total number counted in the sections per brain was multiplied by 5 to estimate the total number of positive cells in the SGZ of the hippocampus.

Statistical analysis. All the data are presented as the means \pm SEM. The following statistical analyses were performed using SPSS (version 17.0; SPSS, Inc., Chicago, IL, USA): one-way analysis of variance (ANOVA) with a post hoc Bonferroni test was used to compare the level of survivin mRNA and protein collected at different time points following TBI. A Student's t test was used to analyze the number of survivin (+), BrdU (+), DCX (+), BrdU/survivin (+) and DCX/survivin (+) cells between the sham-operated group and the TBI group. A p-value $<0.05$ was considered to indicate a statistically significant difference.

\section{Results}

Increased expression of survivin in hippocampus following $T B I$. To investigate the role of survivin in adult neurogenesis 

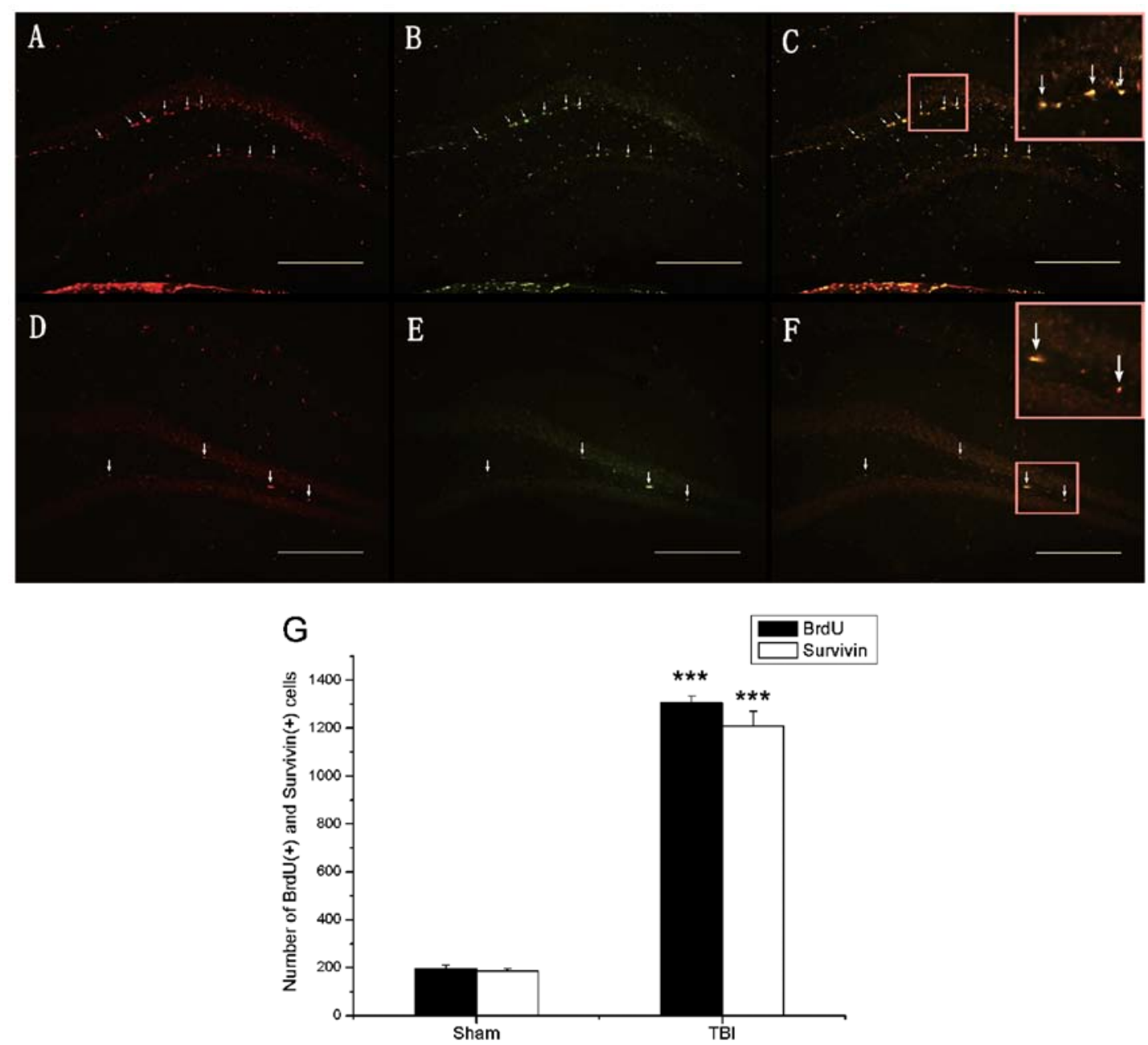

Figure 3. Expression of survivin and proliferation of neural stem cells induced by traumatic brain injury (TBI). (A and B) Double fluorescent staining for survivin (red) and BrdU (green) in the mouse dentate gyrus (DG) of the ipsilateral hippocampus on day 3 post TBI. (C) Merged image of (A) and (B). (D and E) Double fluorescence staining for survivin (red) and BrdU (green) in the DG of the ipsilateral hippocampus in the sham-operated group. (F) Merged image of (D) and (E). Scale bar, $200 \mu \mathrm{m}$. (G) Quantitative analysis of the extent of survivin expression and cell proliferation in the SGZ of the DG. Compared with the sham-operated group, the number of survivin $(+)$ and BrdU $(+)$ cells in the ipsilateral hippocampus was significantly higher in the TBI group $\left(\mathrm{n}=4,{ }^{* * * *} \mathrm{p}<0.001\right)$.

following TBI, we initially examined changes in survivin expression in the ipsilateral hippocampus at the mRNA and protein level. Quantitative PCR analysis revealed that survivin expression was present $12 \mathrm{~h}$ following TBI and was sustained until day 5, with a peak on day 1 and then declined to the level of the sham-operated group on day 14 . The results from quantitative PCR on day 1 in the TBI group revealed a 2-fold increase in expression as compared with the sham-operated group (Fig. 1). These results are in accordance with those from a previous study on rat brains (33). Furthermore, in our study, quantitative western blot analysis was performed to observe the protein expression of survivin following TBI compared with the sham-operated group at each time point; the results revealed that the upregulation of survivin began on day 1 and lasted until day 7, peaking on day 2 (Fig. 2). To further identify the expression of survivin, we performed immunofluorescence staining on day 3 post injury, when survivin protein was steadily expressed following TBI. Compared with the shamoperated group, the number of survivin (+) cells in the SGZ of the DG was significantly increased on day 3 following TBI

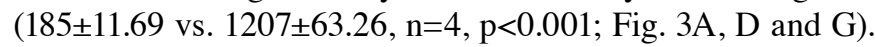

These results strongly suggested that survivin expression was stimulated by brain injury and expressed in a time-dependant manner, with a highest increase 2-5 days following TBI in the hippocampus.

Survivin is expressed in neural stem cells, immature neurons and in a minority of astrocytes, but not in mature neurons in the adult DG of the hippocampus following TBI. We utilized a double staining procedure using BrdU/DCX/GFAP/NeuN and survivin to determine which cell types express high levels of survivin following TBI. We initially found that survivin was expressed by cells in the SGZ of the DG. This suggested that the cells of survivin immunoreactivity in the SGZ may be stem cells and immature neurons. Accordingly, we first observed the BrdU/survivin co-labeled cells in the SGZ of the DG $(93.82 \pm 3 \%, n=4$, Figs. 3C and 7A), suggesting that survivin protein is expressed in the majority of BrdU (+) cells following TBI. We also first found DCX/survivin co-labeled cells in the SGZ of the DG $(61.28 \pm 4 \%, n=4$, Figs. 4C and 7B), indicating that survivin protein is expressed in DCX (+)cells following TBI. The results from immunofluorescence double 

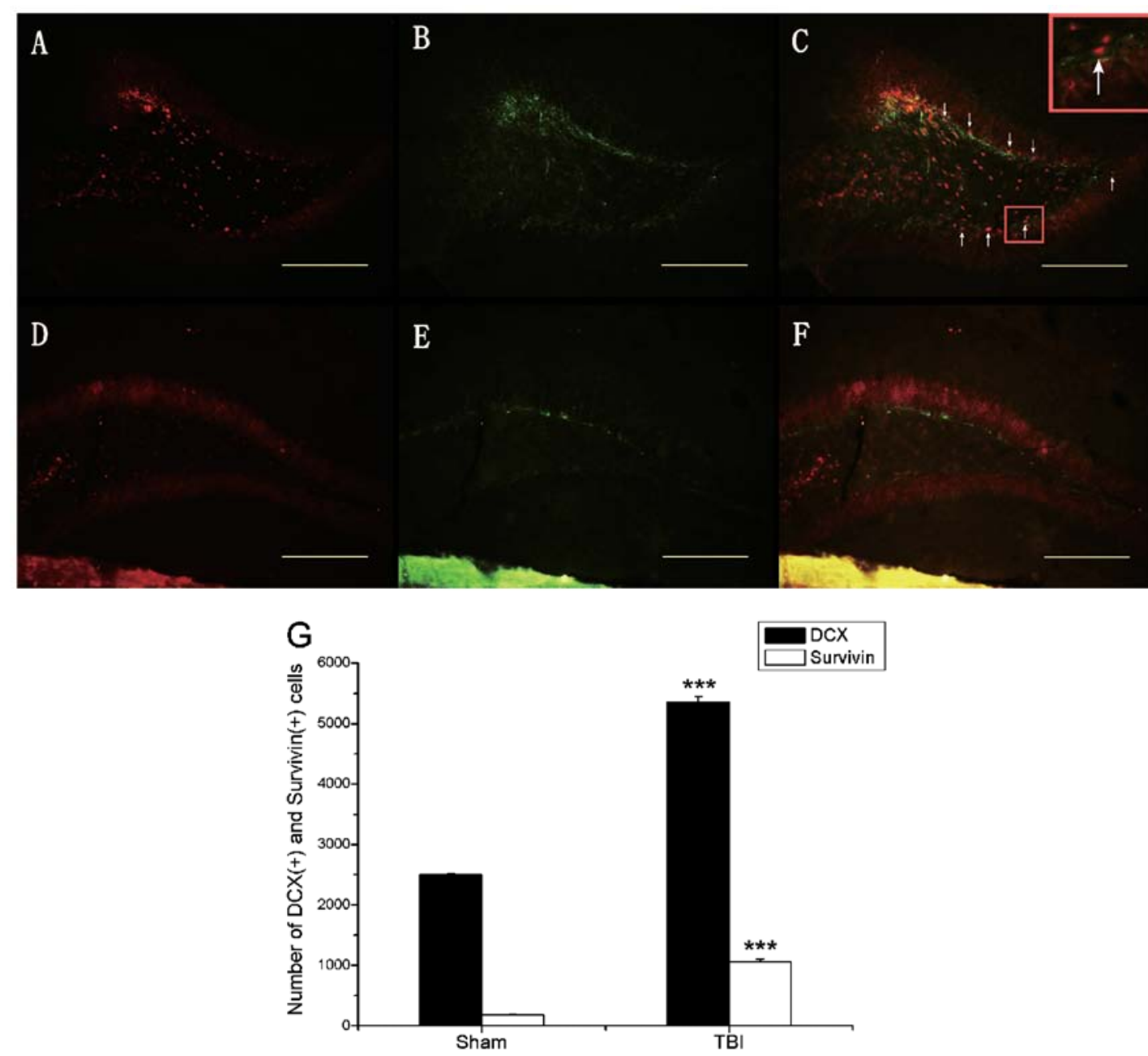

Figure 4. Expression of survivin and increase of immature neurons after traumatic brain injury (TBI). (A and B) Double fluorescence staining for survivin (red) and DCX (green) in the dentate gyrus (DG) of the ipsilateral hippocampus in mouse 3-day after TBI. (C) Merged images of (A) and (B). (D and E) Double fluorescence staining for survivin (red) and DCX (green) in the DG of the ipsilateral hippocampus in the sham-operated group. (F) Merged image of (D) and (E). Scale bar, $200 \mu \mathrm{m}$. (G) Quantitative analysis of the extent of survivin expression and immature neuron increase in the subgranular zone (SGZ) of the DG. Compared to the sham-operated group, the number of survivin $(+)$ and DCX $(+)$ cells in the ipsilateral hippocampus was significantly higher in the TBI group $\left(\mathrm{n}=4,{ }^{* * * *} \mathrm{p}<0.001\right)$.

staining clearly demonstrated that increased levels of survivin expression following TBI were mainly found in stem cells and immature neurons in the SGZ of the DG. In addition, as shown in Figs. 5 and 6, survivin protein expression was observed in a minority of GFAP $(+)$ cells, but not in $\mathrm{NeuN}(+)$ cells, indicating that increased survivin expression is partly observed in astrocytes and not in mature neurons soon after TBI. The model of cell count statistics is presented in Fig. 7.

We found that survivin protein was expressed in the majority of BrdU (+) and DCX (+) cells and in the minority of GFAP (+) cells, but not in NeuN (+) cells post injury. This strongly suggests that increased survivin expression is mainly observed in neural stem cells and immature neurons.

Increased expression of survivin correlates with the proliferation of neural stem cells in the DG of the hippocampus soon after TBI. Quantitative western blot analysis revealed that survivin was steadily expressed on day 3 following TBI. To confirm the correlation between survivin expression and the proliferation of neural stem cell after injury, we quantified the number of survivin $(+)$ and BrdU (+) cells on day 3 following TBI by immunofluorescence staining. When comparing the extent of cell proliferation between the sham-operated group and the TBI group, the number of BrdU (+) cells in the DG was significantly higher than that in the TBI group $(196 \pm 13.5$ vs. $1306 \pm 28.36, n=4, p<0.001$; Fig. 3B, E and G). Compared with the sham-operated group, the number of survivin $(+)$ cells increased in the DG of the hippocampus in the TBI group $(185 \pm 11.69$ vs. $1207 \pm 63.26, n=4, p<0.001$; Figs. 3A, D and $G)$. Quantitative analysis revealed a high percentage of BrdU/survivin co-labeled cells in the TBI group (93.82 $\pm 3 \%$, $\mathrm{n}=4$; Figs. 3C and 7A) and the sham-operated group $(96.42 \pm 1 \%$, $\mathrm{n}=4$; Fig. 3F). These results strongly suggest that the increased expression of survivin promotes the proliferation of neural stem cells in the DG of the hippocampus soon after TBI.

Significant increase in the expression of survivin in DCX (+) immature neurons in the DG of the hippocampus soon after TBI. As described above, survivin promotes hippocampal neurogenesis following TBI. To determine whether survivin is involved in mediating the process of differerentiation in hippocampal neurogenesis following injury, we first measured the expression of survivin in DCX (+) cells in the SGZ of the DG. The immunofluorescence double staining revealed that 


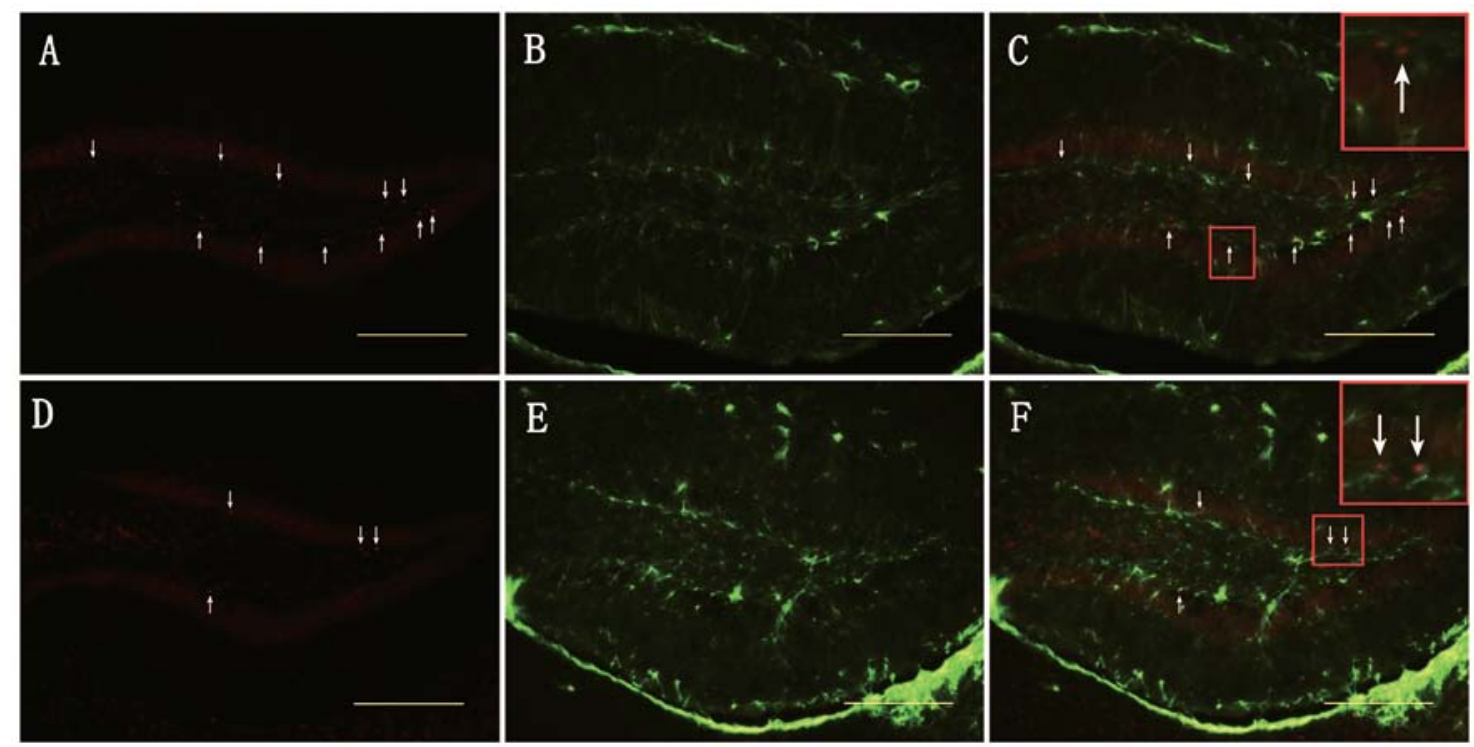

Figure 5. Expression of survivin in astrocytes after traumatic brain injury (TBI). (A and B) Double fluorescence staining for survivin (red) and GFAP (green) in the dentate gyrus (DG) of the ipsilateral hippocampus on day 3 post TBI. (C) Merged images of (A) and (B). (D and E) Double fluorescence staining for survivin (red) and GFAP (green) in the DG of the ipsilateral hippocampus in the sham-operated group. (F) Merged images of (D) and (E). Scale bar, $200 \mu \mathrm{m}$.
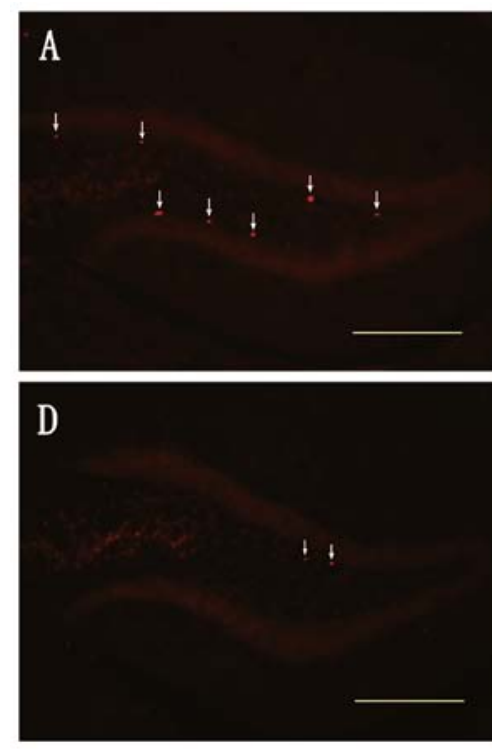
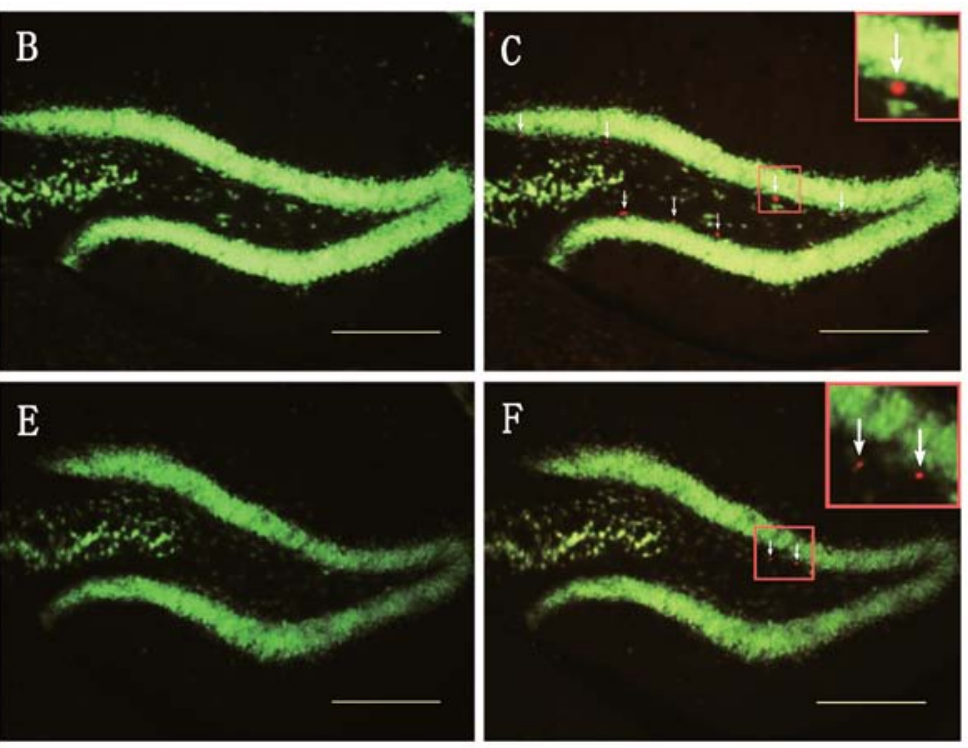

Figure 6. Expression of survivin in mature neurons after traumatic brain injury (TBI). (A and B) Double fluorescence staining for survivin (red) and NeuN (green) in the dentate gyrus (DG) of the ipsilateral hippocampus on day 3 post TBI. (C) Merged images of (A) and (B). (D and E) Double fluorescence staining for survivin (red) and NeuN (green) in the DG of the ipsilateral hippocampus in the sham-operated group. (F) Merged images of (D) and (E). Scale bar, $200 \mu \mathrm{m}$.

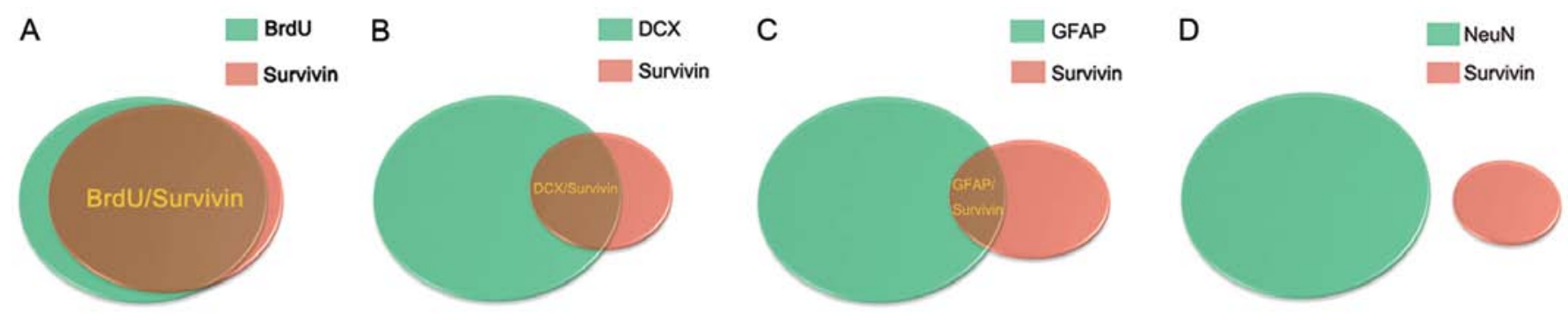

Figure 7. Model of different cell types expressing survivin in the subgranular zone (SGZ) of the hippocampus after traumatic brain injury (TBI). (A) Almost all of survivin protein (red) is expressed in the majority of BrdU (+) cells (green) after TBI. (B) Part of survivin protein (red) is expressed in many DCX (+) cells (green) after TBI. (C) Little survivin protein (red) is associated with the minority of GFAP (+) cells (green) after TBI. (D) No NeuN (+) cells (green) expressed survivin protein (red). 
the number of survivin $(+)$ cells and DCX-positive cells was increased in the TBI group compared with the sham-operated group (55 \pm 8.21 vs. $646 \pm 30.03$, n=4, p<0.001; Fig. 4C, F and G) A significant percentage of survivin (+) cells also expressed DCX $(61.28 \pm 4 \%, n=4$; Figs. $4 C$ and $7 B)$ indicating that the expression of survivin was increased in immature neurons in the DG of the hippocampus. This suggests that survivin plays a role in the survival of immature neurons in the DG of the hippocampus soon after TBI and promotes the differentiation of these immature neurons into granular neurons.

\section{Discussion}

It is well known that adult neurogenesis exists in the CNS. New neurons are continuously generated from neural stem/progenitor cells in the SGZ of the DG throughout adulthood (34). Previous studies have demonstrated the correlation between the Wnt/ $\beta$-catenin signaling pathway and adult neurogenesis. In the adult brain, stabilized $\beta$-catenin modulates the proliferation of neural stem cells, induces newly generated neuronal cell migration into relevant regions and then mediates their differentiation into mature neurons and ultimately plays a role in synaptogenesis (14). The disruption of signaling is associated with several pathological diseases, such as Alzheimer's disease (AD) (35-37), schizophrenia (38) and autism (39).

Survivin, as the downstream targetgene of the Wnt/ $\beta$-catenin signaling pathway $(16,17)$, plays a critical role in protecting neuronal cells from apoptosis and promoting the proliferation of neurogenic progenitor cells within the developing CNS. When survivin expression is disrupted in the developing brain, embryos survive until birth, but the majority of the brain is hypoplastic. On a cellular level, this phenotype occurs as a result of massive neuronal apoptosis. Survivin has been linked to the inhibition of cell apoptosis in developing tissues, but the exact molecular mechanisms involved remain unclear. Studies have shown that the activity of caspase- 3 and caspase- 9 in the survivin-null brains is increased, supporting the role of survivin in inhibiting caspase- 3 and caspase- 9 activity, thus preventing apoptosis, through either indirect or direct mechanisms (26). Survivin plays a role in mitosis as a CPP $(40,41)$. In this capacity, it recruits aurora B kinase to the CPP complex and ensures the proper alignment of chromosomes before cell division (42). The majority of survivin ${ }^{-/}$embryos have grossly enlarged nuclei with abnormal morphology. As this phenotype progresses, the cells cease to complete mitosis, with the decreasing number of normal cells replaced by a small number of giant cells with large and morphologically unusual nuclei. Overall, survivin is an essential factor during the development of the mammalian nervous system.

Survivin is prominently expressed in the neurogenic regions of the embryonic $\mathrm{CNS}$ and its expression is maintained by a subpopulation of neural progenitor cells post-natally in the 2 key sites of adult neurogenesis, the SVZ and the SGZ (43). In our study, we investigated the correlation between survivin expression and adult neurogenesis following TBI in the DG of the hippocampus. Our results revealed that the expression of survivin was increased in the mouse hippocampus in a time-dependent manner and this change in expression was associated with the proliferation of stem cells early following TBI. Johnson et al detected changes in survivin expression after injury in the rat brain (30), suggesting the correlation between survivin and neurogenesis induced by TBI. To further demonstrate the role of survivin expression in neurogenesis following TBI, we utilized a double staining procedure of BrdU/DCX/GFAP/NeuN and survivin to observe cell types with an increased expressio of survivin following TBI. According to previous studies reporting the PCNA/survivin co-labeled cells (33), we first observed the BrdU/survivin co-labeled cells in the SGZ of the DG, suggesting that survivin is expressed in neural stem cells. The results also revealed that survivin is expressed in immature neurons and in a minority of astrocytes, but not in mature neurons in the adult DG of the hippocampus following TBI. Through the use of immunofluorescence staining, newly generated cells labeled with BrdU were detected and the majority of BrdU-labeled cells merged with survivin (+) cells in the DG. The number of BrdU (+) and survivin (+) cells increased in the TBI group versus the sham-operated group. These results strongly suggest that TBI-induced neurogenesis may be associated with the upregulation of survivin expression and that survivin plays a role in promoting the proliferation of neural stem cell following injury. Moreover, the double staining of survivin and DCX revealed that a significant percentage of DCX (+) cells also expressed survivin in the TBI group compared with the sham- operated group, which indicated that the expression of survivin was increased in immature neurons in the DG of the hippocampus sooon after TBI. We consider that the expression of survivin is critical to the proliferation of neural stem cells and to the survival of existing cells in CNS development, as well as to the functional maintenance of the adult brain. Furthermore, survivin may play a role in the repair and restoration of neurons in certain pathological processes, such as TBI.

Injury-induced neurogenesis is a compelling potential contributor to recovery post-injury $(7,8,44)$. Progenitor cells in the SGZ are activated following TBI, although it remains unclear whether this activation results in stable and productive neurogenesis (8). In addition, evidence for long-lasting hippocampal neurogenesis after traumatic cortical injury has been accumulating (10). Even in adult animals, it is clear that progenitors are activated and neurogenesis increases following injury, whereby new neurons are found in the outer layers of the DG $(45,46)$.

The majority of the evidence to support these findings is based on the increased dividing cells labeled by BrdU when the proliferation of stem cells/progenitors is produced following brain injury. The DCX-expressing cells within the DG re-emerge and are the likely contributors to stable neurogenesis (47). DCX, a marker of developing, immature neurons, has been found in the adult rostal migratory stream and in the DG of the hippocampus in adult rats during the early differentiation stage of adult neurogenesis (48). DCX (+) cells have also been shown to be present near and among the glial scars following brain injury (49). Similarly, our study showed an increase in the number of BrdU (+) and DCX (+) cells in the SGZ, also suggesting that the proliferation of stem cells/progenitors was induced by injury. On the contrary, Rola et al found that DCX (+) and BrdU (+) cells were reduced in the ipsilateral SGZ following brain injury (50). This discrepancy may be caused by the different experimental methods and animal conditions, i.e., the model established, degree of injury, 
housing conditions and technical considerations. However, it is important to note that BrdU is not a marker of the $\mathrm{S}$ phase of the cell cycle; it is a marker of DNA synthesis. Therefore, it also labels cells undergoing DNA repair, aborting cell cycle re-entry, as a prelude to apoptosis (51). However, the majority of BrdU (+) cells were proliferating cells. Barha et al showed a high percentage of BrdU (+) cells co-expressing DCX after TBI (89.00\% \pm 3.42$)(52)$ and a co-localization of BrdU and DCX represents stem cells soon after injury, suggesting that the majority of the BrdU (+) cells were the proliferating stem cells in the SGZ.

Yu et al observed that DCX-expressing neural progenitors are required for injury-induced remodeling to occur in a highly proliferative environment, as evidenced by BrdU pulsing (47) and we demonstrated the increased expression of survivin in DCX (+) cells, suggesting the crucial role of survivin in neurogenesis induced by injury. However, there was a discrepancy as regards the time point in DCX increasing expression after injury between our 2 research groups. The potential factors that caused this discrepancy were experimental methods and animal conditions. Furthermore, Yu et al also demonstrated the essential role of nestin-expressing early neural progenitors in TBI-induced hippocampal neurogenesis (47). We consider that survivin is expressed in the nestin-expressing progenitors and may be involved in neurogenesis completed by the activation of nestin-expressing progenitors. Further studies are required to investigate the mechanisms of action of the survivin gene in injury-induced adult neurogenesis, which is dependent on the participation of different phenotypes of stem cells/progenitors, i.e., BrdU (+), DCX (+) and nestin (+) cells. The necessary alteration of survivin gene expression, i.e., downregulation with siRNA or the development of survivin transgenic mice, may aid in providing further insight into its role in neurogenesis.

Strong evidence is accumulating for a role of survivin in certain pathological processes, such as neurodegenerative diseases (3) and brain ischemia (29,53-56). Based on these data, we hypothesized that survivin, a downstream target gene of the $\mathrm{Wnt} / \beta$-catenin signaling pathway, plays a role in repairing deterioration and promoting recovery of neural function in neuronal degeneration diseases and various brain injuries involving ischemia and trauma. However, the mechanisms of TBI require further investigatation. Our study only elucidated the correlation between survivin expression and neurogenesis following TBI and initially explored the function of survivin expression. We found that survivin plays a role in the proliferation and differentiation of neural cells following injury. Further investigation is required to further elucidate its role in neurogenesis, in order to provide basic methods of treatment for brain injury and recovery of dysfunction.

\section{Acknowledgements}

We thank Linchun Huan, Wangmiao Zhao and Zhen Zhang for their helpful comments on the manuscript. This study was supported by grants from the Science and Technology Foundation of Tianjin (no. 10JCYBJC25700) and the Health-System Science Foundation of Binhai New Area in Tianjin (no. 2011BHKL002).

\section{References}

1. Narayan RK, Michel ME, Ansell B, et al: Clinical trials in head injury. J Neurotrauma 19: 503-557, 2002.

2. Pickard JD and Czosnyka M: Management of raised intracranial pressure. J Neurol Neurosurg Psychiatry 56: 845-858, 1993.

3. Baratchi S, Kanwar RK and Kanwar JR: Survivin: a target from brain cancer to neurodegenerative disease. Crit Rev Biochem Mol Biol 45: 535-554, 2010.

4. Richardson RM, Singh A, Sun D, Fillmore HL, Dietrich DW III and Bullock MR: Stem cell biology in traumatic brain injury: effects of injury and strategies for repair. J Neurosurg 112: $1125-1138,2010$.

5. Alvarez-Buylla A and Lim DA: For the long run: maintaining germinal niches in the adult brain. Neuron 41: 683-686, 2004.

6. Lie DC, Song H, Colamarino SA, Ming GL and Gage FH: Neurogenesis in the adult brain: new strategies for central nervous system diseases. Annu Rev Pharmacol Toxicol 44: 399-421, 2004

7. Chirumamilla S, Sun D, Bullock MR and Colello RJ: Traumatic brain injury induced cell proliferation in the adult mammalian central nervous system. J Neurotrauma 19: 693-703, 2002.

8. Richardson RM, Sun D and Bullock MR: Neurogenesis after traumatic brain injury. Neurosurg Clin N Am 18: 169-181, 2007.

9. Zhao WM, Huan LC, Zhao Y, et al: Endogenous adult neurogenesis and cognitive function recovery following traumatic brain injury in the rat hippocampus. Neural Regen Res 5: 645-650, 2010.

10. Dash PK, Mach SA and Moore AN: Enhanced neurogenesis in the rodent hippocampus following traumatic brain injury. J Neurosci Res 63: 313-319, 2001.

11. Sun D, Colello RJ, Daugherty WP, Kwon TH, McGinn MJ, Harvey HB and Bullock MR: Cell proliferation and neuronal differentiation in the dentate gyrus in juvenile and adult rats following traumatic brain injury. J Neurotrauma 22: 95-105, 2005.

12. Gulacsi AA and Anderson SA: Beta-catenin-mediated Wnt signaling regulates neurogenesis in the ventral telencephalon. Nat Neurosci 11: 1383-1391, 2008.

13. Zechner D, Fujita Y, Hulsken J, et al: beta-Catenin signals regulate cell growth and the balance between progenitor cell expansion and differentiation in the nervous system. Dev Biol 258: 406-418, 2003.

14. Zhang L, Yang X, Yang S and Zhang J: The Wnt/beta-catenin signaling pathway in the adult neurogenesis. Euro J Neurosci 33: $1-8,2011$.

15. White BD, Nathe RJ, Maris DO, Nguyen NK, Goodson JM, Moon RT and Horner PJ: Beta-catenin signaling increases in proliferating NG2+ progenitors and astrocytes during posttraumatic gliogenesis in the adult brain. Stem Cells 28: 297-307, 2010.

16. Zhang T, Otevrel T, Gao Z, Ehrlich SM, Fields JZ and Boman BM: Evidence that APC regulates survivin expression: a possible mechanism contributing to the stem cell origin of colon cancer. Cancer Res 61: 8664-8667, 2001.

17. Zhu H, Zhang G, Wang Y, et al: Inhibition of ErbB2 by Herceptin reduces survivin expression via the ErbB2-beta-catenin/ TCF4-survivin pathway in ErbB2-overexpressed breast cancer cells. Cancer Sci 101: 1156-1162, 2010.

18. Deveraux QL and Reed JC: IAP family proteins-suppressors of apoptosis. Genes Dev 13: 239-252, 1999.

19. Li F, Ambrosini G, Chu EY, Plescia J, Tognin S, Marchisio PC and Altieri DC: Control of apoptosis and mitotic spindle checkpoint by survivin. Nature 396: 580-584, 1998.

20. Hirokawa N: Microtubule organization and dynamics dependent on microtubule-associated proteins. Curr Opin Cell Biol 6: 74-81, 1994.

21. Altieri DC: Survivin, versatile modulation of cell division and apoptosis in cancer. Oncogene 22: 8581-8589, 2003.

22. Kobayashi K, Hatano M, Otaki M, Ogasawara T and Tokuhisa T: Expression of a murine homologue of the inhibitor of apoptosis protein is related to cell proliferation. Proc Natl Acad Sci USA 96: 1457-1462, 1999.

23. O'Connor DS, Schechner JS, Adida C, et al: Control of apoptosis during angiogenesis by survivin expression in endothelial cells. Am J Pathol 156: 393-398, 2000.

24. Shin S, Sung BJ, Cho YS, et al: An anti-apoptotic protein human survivin is a direct inhibitor of caspase-3 and -7. Biochemistry 40: 1117-1123, 2001. 
25. Tamm I, Wang Y, Sausville E, Scudiero DA, Vigna N, Oltersdorf T and Reed JC: IAP-family protein survivin inhibits caspase activity and apoptosis induced by Fas (CD95), Bax, caspases and anticancer drugs. Cancer Res 58: 5315-5320, 1998.

26. Jiang Y, de Bruin A, Caldas H, et al: Essential role for survivin in early brain development. J Neurosci 25: 6962-6970, 2005.

27. Zwerts F, Lupu F, De Vriese A, et al: Lack of endothelial cell survivin causes embryonic defects in angiogenesis, cardiogenesis, and neural tube closure. Blood 109: 4742-4752, 2007.

28. Adida C, Crotty PL, McGrath J, Berrebi D, Diebold J and Altieri DC: Developmentally regulated expression of the novel cancer anti-apoptosis gene survivin in human, and mouse differentiation. Am J Pathol 152: 43-49, 1998

29. Li F and Brattain MG: Role of the Survivin gene in pathophysiology. Am J Pathol 169: 1-11, 2006.

30. Johnson EA, Svetlov SI, Wang KK, Hayes RL and Pineda JA Cell-specific DNA fragmentation may be attenuated by a survivin-dependent mechanism after traumatic brain injury in rats. Exp Brain Res 167: 17-26, 2005.

31. Thompson HJ, Lifshitz J, Marklund N, Grady MS, Graham DI, Hovda DA and McIntosh TK: Lateral fluid percussion brain injury: a 15-year review and evaluation. J Neurotrauma 22: 42-75, 2005 .

32. Wojtowicz JM and Kee N: BrdU assay for neurogenesis in rodents. Nat Protoc 1: 1399-1405, 2006

33. Johnson EA, Svetlov SI, Pike BR, et al: Cell-specific upregulation of survivin after experimental traumatic brain injury in rats. J Neurotrauma 21: 1183-1195, 2004.

34. Ray J, Peterson DA, Schinstine M and Gage FH: Proliferation, differentiation, and long-term culture of primary hippocampal neurons. Proc Natl Acad Sci USA 90: 3602-3606, 1993.

35. De Ferrari GV and Inestrosa NC: Wnt signaling function in Alzheimer's disease. Brain Res Brain Res Rev 33: 1-12, 2000.

36. De Ferrari GV, Papassotiropoulos A, Biechele T, et al: Common genetic variation within the low-density lipoprotein receptorrelated protein 6 and late-onset Alzheimer's disease. Proc Natl Acad Sci USA 104: 9434-9439, 2007.

37. Inestrosa NC, Alvarez A, Godoy J, Reyes A and De Ferrari GV: Acetylcholinesterase-amyloid-beta-peptide interaction and Wnt signaling involvement in Abeta neurotoxicity. Acta Neurol Scand Suppl 176: 53-59, 2000

38. Lovestone S, Killick R, Di Forti M and Murray R: Schizophrenia as a GSK-3 dysregulation disorder. Trends Neurosci 30: 142-149, 2007.

39. De Ferrari GV and Moon RT: The ups and downs of Wnt signaling in prevalent neurological disorders. Oncogene 25 : 7545-7553, 2006

40. Wheatley SP, Carvalho A, Vagnarelli P and Earnshaw WC INCENP is required for proper targeting of Survivin to the centromeres and the anaphase spindle during mitosis. Curr Biol 11: 886-890, 2001.

41. Skoufias DA, Mollinari C, Lacroix FB and Margolis RL: Human survivin is a kinetochore-associated passenger protein. J Cell Biol 151: 1575-1582, 2000

42. Chen J, Jin S, Tahir SK, et al: Survivin enhances Aurora-B kinase activity and localizes Aurora-B in human cells. J Biol Chem 278: 486-490, 2003.
43. Coremans V, Ahmed T, Balschun D, et al: Impaired neurogenesis, learning and memory and low seizure threshold associated with loss of neural precursor cell survivin. BMC Neurosci 11: 2, 2010.

44. Kernie SG and Parent JM: Forebrain neurogenesis after focal Ischemic and traumatic brain injury. Neurobiol Dis 37: 267-274, 2010.

45. Ramaswamy S, Goings GE, Soderstrom KE, Szele FG and Kozlowski DA: Cellular proliferation and migration following a controlled cortical impact in the mouse. Brain Res 1053: 38-53, 2005.

46. Urrea C, Castellanos DA, Sagen J, Tsoulfas P, Bramlett HM and Dietrich WD: Widespread cellular proliferation and focal neurogenesis after traumatic brain injury in the rat. Restor Neurol Neurosci 25: 65-76, 2007.

47. Yu TS, Zhang G, Liebl DJ and Kernie SG: Traumatic brain injury-induced hippocampal neurogenesis requires activation of early nestin-expressing progenitors. J Neurosci 28: 12901-12912, 2008.

48. Brown JP, Couillard-Despres S, Cooper-Kuhn CM, Winkler J, Aigner L and Kuhn HG: Transient expression of doublecortin during adult neurogenesis. J Comp Neurol 467: 1-10, 2003.

49. Itoh T, Satou T, Nishida S, Hashimoto $S$ and Ito H: Immature and mature neurons coexist among glial scars after rat traumatic brain injury. Neurol Res 29: 734-742, 2007.

50. Rola R, Mizumatsu S, Otsuka S, Morhardt DR, NobleHaeusslein LJ, et al: Alterations in hippocampal neurogenesis following traumatic brain injury in mice. Exp Neurol 202: 189-199, 2006.

51. Taupin P: BrdU immunohistochemistry for studying adult neurogenesis: paradigms, pitfalls, limitations, and validation. Brain Res Rev 53: 198-214, 2007.

52. Barha CK, Ishrat T,Epp JR, Galea LA and Stein DG: Progesterone treatment normalizes the levels of cell proliferation and cell death in the dentate gyrus of the hippocampus after traumatic brain injury. Exp Neurol 231: 72-81, 2011

53. Conway EM, Zwerts F, Van Eygen V, DeVriese A, Nagai N, Luo W and Collen D: Survivin-dependent angiogenesis in ischemic brain: molecular mechanisms of hypoxia-induced up-regulation. Am J Pathol 163: 935-946, 2003.

54. Lu N, Li XJ, Li CZ, Li DL, Cui PF, Hou YN and Wang YL: Effect of hypoxic preconditioning on the learning and memory ability and expressions of survivin and HSP-70 proteins in rats with focal cerebral ischemia/reperfusion injury. Nan Fang Yi Ke Da Xue Xue Bao 27: 1856-1859, 2007 (Article in Chinese).

55. Okazaki T, Magaki T, Takeda M, et al: Intravenous administration of bone marrow stromal cells increases survivin and Bcl-2 protein expression and improves sensorimotor function following ischemia in rats. Neurosci Lett 430: 109-114, 2008.

56. Zhang Y, Park TS and Gidday JM: Hypoxic preconditioning protects human brain endothelium from ischemic apoptosis by Akt-dependent survivin activation. Am J Physiol Heart Circ Physiol 292: H2573-H2581, 2007. 